\title{
Single-qubit lasing in the strong-coupling regime
}

Authors: Stephan André, Pei-Qing Jin, Valentina Brosco, Jared H. Cole, Alsessandro Romito, Alexander Shnirman, and Gerd Schön

arXiv:1008.2611

Recommended and Commentary by Steven M. Girvin, Yale University

The experimental development of quantum optics of electrical circuits continues at a rapid pace. Artificial atoms using Josephson junctions are now routinely engineered to couple extremely strongly to microwave photons trapped in on-chip resonators. In fact, because the 'atoms' are large and the cavities are small, the coupling can reach the limit set by the fine-structure constant [1]. As I described previously (http://www.condmatjournalclub.org/?p=530), Astafiev et al. [2] achieved single-atom lasing using a superconducting singleelectron transistor device coupled to a resonator.

Now André et al. have developed a theory of single-qubit lasing in the strong-coupling regime. This regime of small atom number and strong coupling is very different [3] from that historically considered in lasing theory. Here quantum noise dominates over thermal fluctuations and the usual simple phase-diffusion model of the laser spectral output does not apply.

Laser light (or in this case, microwaves) paradoxically is classical, or at least as classical as any light can be. It has the same properties as the output of a classical radio station. The electric field oscillates sinusoidally in time with constant amplitude, but slow random phase fluctuations. Fourier transform gives a Lorentzian spectral line width which is determined by the inverse of the time it takes the phase to random walk through an angle of order $\pi$. The only quantum mechanics we need to remember is that a photomultiplier detects the energy in the wave in discrete photon 'lumps.' In a laser beam these lumps are randomly distributed and completely uncorrelated with each other. It is interesting to compare this to the output of a thermal black-body source. By passing the light through a suitable filter it can be made to have exactly the same spectrum as our laser. The difference however can be found in the fact the thermal beam has both amplitude and phase fluctuations. The amplitude fluctuations lead to positive correlations in the positions of the photons. This 'photon bunching' is determined by a higher-order field correlation function, $g_{2}$, which distinguishes the thermal source from the laser, even when their spectra are identical. Recent developments have brought measurement of this and related higher-order correlation 
functions within reach for microwave photons [4-9].

Imagine now another extreme limit, of resonance fluorescence of a single atom weakly driven by a laser. If the atom emits a fluorescence photon then we know it is in the ground state and there will be some time delay before the atom is re-pumped to the excited state where it can emit the next photon. Hence the light output has the photons anti-bunched. In this limit [3], it is not clear that we should think of the single-atom laser as a laser at all.

In this paper and its predecessors in the series, André et al. have developed two different analytical and numerical techniques for dealing with the quantum fluctuations that dominate this interesting strong-correlation regime of small atom number and large atom-photon coupling.

\section{References:}

1. 'Wiring up quantum systems,' R.J. Schoelkopf and S.M. Girvin, 'Horizons' Review, Nature 451, 664 (2008).

2. 'Single artificial-atom lasing,' O. Astafiev, K. Inomata, A.O. Niskanen, T. Yamamoto, Yu. A. Pashkin, Y. Nakamura, and J.S. Tsai, Nature 449, 588 (2007).

3. 'Experimental realization of a one-atom laser in the regime of strong coupling,' J. McKeever, A. Boca, A. D. Boozer, J. R. Buck, and H. J. Kimble, Nature 425, 268-271 (2003).

4. 'Environmental effects in the third moment of voltage fluctuations in a tunnel junction,' B. Reulet, J. Senzier, D.E. Prober, Phys. Rev. Lett. 91, 196601 (2003).

5. 'The third moment of current fluctuations in a tunnel junction: experiments in the classical and quantum regimes,' B. Reulet, J. Gabelli, L. Spietz, D.E. Prober, arXiv:1001.3034.

6. 'Schemes for the observation of photon correlation functions in circuit QED with linear detectors,' Marcus P. da Silva, Deniz Bozyigit, Andreas Wallraff, Alexandre Blais, arXiv:1004.3987.

7. 'Measurements of the Correlation Function of a Microwave Frequency Single Photon Source,' D. Bozyigit, C. Lang, L. Steffen, J. M. Fink, M. Baur, R. Bianchetti, P. J. Leek, S. Filipp, M. P. da Silva, A. Blais, A. Wallraff, arXiv:1002.3738. 
8. 'Dual-path state reconstruction scheme for propagating quantum microwaves and detector noise tomography,' E. P. Menzel, F. Deppe, M. Mariantoni, M. A. A. Caballero, A. Baust, T. Niemczyk, E. Hoffmann, A. Marx, E. Solano, and R. Gross (2010), arXiv:1001.3669.

9. 'Planck Spectroscopy and the Quantum Noise of Microwave Beam Splitters,' M. Mariantoni, E. P. Menzel, F. Deppe, M. A. A. Caballero, A. Baust, T. Niemczyk, E. Hoffmann, E. Solano, A. Marx, and R. Gross (2010), arXiv:1003.3194. 